\title{
Ultracold Alkali Atoms in Optical Lattices: A New Type of Quantum Crystals?
}

\author{
Alexander E. Meyerovich \\ Department of Physics, University of Rhode Island, \\ Kingston, RI 02881, USA
}

Similarities between alkali gases in optical lattices with non-integer occupation of the lattice sites and quantum crystals are explored. The analogy with the vacancy liquid provides an alternative explanation to the Mott transition for the recent experiment on the phase transition in the lattice. The vacancy liquid can undergo BEC with $T_{c}$ within experimental reach. Direct and vacancy-assisted mechanisms of the band motion for hyperfine impurities are discussed. The presence of vacancies can result in the spatial decomposition of the system into pure hyperfine components. Below BEC for the vacancies, the impurity component resembles ${ }^{3} \mathrm{He}$ in ${ }^{3} \mathrm{He}-\mathrm{HeII}$ mixtures. PACS numbers: 03.75.Fi, 05.30.Jp, 66.35.+a, 67.80.-s.

\section{INTRODUCTION}

Experimental discovery of Bose condensation made the study of ultracold alkali gases a focal point in atomic, low temperature, and condensed matter physics. Experiments revealed some of the phenomena that have been discussed earlier only within theoretical models ${ }^{1}$ and that combine features inherent to diverse condense matter and low temperature systems ${ }^{2}$. For example, BEC in trapped gases resembles, but is not quite the same as the transition in other superfluid systems. Another example is the dynamics of the hyperfine components which resembles the spin dynamics in spin-polarized quantum gases ${ }^{3}$.

One more example is an ultracold alkali gas in an optical lattice in which the atoms are located in periodic potential wells induced by the Stark effect of interfering laser beams ${ }^{4}$. The depth $V_{0}$ and width $a$ of the wells and the rate of tunneling between them are determined by the intensity 


\section{A. E. Meyerovich}

and the wavelength $\lambda$ of the beams $(a=\lambda / 2=\pi / k)$. By adjusting the intensity, one can scan a wide range of the tunneling frequency $t$, effective mass $m^{*} \sim \hbar^{2} / t a^{2}$, and repulsion $U$ of atoms inside the same well from a free gas with a periodic perturbation to a well-localized "solid".

According to the Hubbard model, the system undergoes ${ }^{5}$ the Mott metal-insulator transition at $U / t \approx 5.8 z$ ( $z$ is the number of the nearest neighbors). At $U \gg t$, the system is an "insulator" without interwell transitions (the tunneling increases the on-site energy by $U$ and is energetically prohibitive). At $U \ll t$, the interaction does not restrict tunneling and the atoms are in the "metal" phase. At low temperature, such "metal" undergoes BEC into a lattice superfluid. The energies $U, t$, and $V_{0}$ are often measured in units of the recoil energy $E_{r}=\hbar^{2} k^{2} / 2 m$. A typical example ${ }^{5}$ is $t / E_{r} \sim 0.07$ and $U / E_{r} \sim 0.15$ for $V_{0} / E_{r}=15$.

This transition was reported in Ref. 6 for the lattice with $a \sim 426 \mathrm{~nm}$ and $E_{r} \sim 1 \mathrm{kHz}$. At low beam intensity (large $t$ ), there was a BEC peak in the center of the trap. The peak disappeared at small $t\left(V_{0} / E_{r}\right.$ between 13 and 22). The identification of this new phase as the Mott insulator (MI) was not direct. The MI exists only when the average population of a lattice site is integer. If the population is fractional, the highest on-site states are not fully occupied. The tunneling of a particle from the occupied to an unoccupied state cannot be banned by the on-site interaction since such tunneling is not energetically prohibitive. This opens the way to the band motion of the "excessive" particles leaving the lattice with non-integer occupation in the "metal" or "semiconductor" state even at large $U$. Though there is experimental evidence ${ }^{6}$ that the conduction band is separated from the filled band by a gap, it is difficult to conclude whether the conduction gap is empty or not. Below we suggest an alternative interpretation for Ref. 6 based on the analogy with the quantum crystals (QC).

\section{VACANCIES AND IMPURITIES IN OPTICAL LATTICES}

There is a similarity between the particles in optical lattices and atoms in $\mathrm{QC}$, such as solid helium, in which the interstate tunneling ensures the band motion of atoms unless prohibited by large on-site repulsion (see review ${ }^{7}$ ). In helium crystals, the atomic band motion is impossible, as for all MI, when the lattice sites are occupied by identical particles with the occupancy equal to one. If some of the lattice sites are empty, nothing prohibits tunneling of atoms from the occupied onto the vacant sites leading to the formation of peculiar band quasiparticles - vacancy waves. Similar quasiparticles are formed when atoms occupy interstitial sites or when some of the lattice sites 


\section{Optical Lattices: A New Type of Quantum Crystals?}

are occupied by atoms of a different kind (impurities). The impurities also tunnel through the QC even if each site has the occupancy equal to one. The tunneling rate for impurities is smaller than for the vacancies since the exchange of places between the impurity and host atoms involves high-energy intermediate states with either double on-site occupancy or the atom in an interstitial position. Often, a more efficient mechanism of impurity motion is the vacancy-assisted diffusion. The vacancy and impurity waves in QC are well understood ${ }^{7}$. However, the most exciting effect - superfluidity and BEC in the system of vacancy waves - has not been observed for "classical" QC, namely, solid ${ }^{4} \mathrm{He}$ despite two decades of intensive efforts ${ }^{8}$. The reason is the absence of the zero-temperature vacancies in ${ }^{4} \mathrm{He}$ : with lowering temperature, the concentration of vacancies drops exponentially always remaining insufficient for BEC.

Atoms in optical lattices resemble QC with an appealing difference: the BEC for vacancy or "impurity" waves could be within reach. When the occupancy of the individual wells is close to an integer $K$, the system resembles QC with either a small density of vacancies $n_{v}=K N-n \ll N$ or "excessive" atoms $n_{e} \equiv N-n_{v}=n-N \operatorname{Int}[n / N] \ll N(n$ and $N$ are the densities of atoms and lattice sites). The tunneling frequencies are the same for vacancies and "excessive" atoms, $t_{v}=t_{e}$, since, in both cases, an atom tunnels to an empty site. As a result, many properties of the system are symmetric with respect to the vacancies and excessive atoms. [In usual QC the environments for a vacancy and an "excessive" atom are different].

Below we consider the situation with large on-site interaction $U$ when the lattice system with the integer site occupation is a Mott insulator.

In the tight binding approximation for vacancies in a cubic lattice, $\epsilon_{v}(\mathbf{p})=\Delta\left(\frac{1}{2}-\frac{1}{6} \sum \cos \left(p_{i} a / \hbar\right)\right)$, where $\Delta=12 t_{v}$ is the bandwidth. The chemical potential $\mu_{v}$ is finite in contrast to $\mu_{v}=0$ for thermally-activated vacancies in helium. When $n_{v}$ (or $n_{e}$ ) is small, the BEC transition temperature in the vacancy gas is determined by the equations for lattice gases with low band filling. A good extrapolation between these limiting cases is

$$
T_{c}=6.6 a^{4} t_{v} n_{v}^{2 / 3}\left(N-n_{v}\right)^{2 / 3} .
$$

With the above values of $E_{r}$ and $a$, the estimate for (1) is $T_{c} \sim 3 \times$ $10^{-7}\left(t_{v} / E_{r}\right) x_{v}^{2 / 3}\left(1-x_{v}\right)^{2 / 3} \mathrm{~K}$ where $x_{v}=a^{3} n_{v}$.

The density of participating particles (vacancies or excessive atoms in the highest on-site state) is lower than the overall density. In the experiment of Ref. 6 with the occupancy between 2 and 3 , this leads to a factor $5^{-2 / 3}$ in $T_{c}$ with respect to a free gas and even stronger lowering of $T_{c}$ in systems close to an integer occupancy. Second, the effective mass $m^{*}=m\left(E_{r} / \pi^{2} t\right)$ could be much larger than the mass of the free atoms $m$. One has limited 


\section{A. E. Meyerovich}

control over the vacancy concentration. On the other hand, the exponential dependence of $m^{*}$ on the intensity of the laser beams can make $T_{c}$ in the vacancy system observable.

This suggests an alternative to the Mott transition for the experiment of Ref. 6. At large $t$, the experiment showed the BEC, probably, in the "free" gas rather than in the vacancy liquid. At small $t$, there was no condensate. However, the experiment, by design, cannot distinguish between the MI and the vacancy liquid. The likely transition was between the superfluid and the vacancy liquid rather than between the superfluid and the MI.

The analogy with QC leads to other predictions. The role of "impurity waves" can be played by atoms in the different hyperfine states which can be studied by means similar to NMR for ${ }^{3} \mathrm{He}$ diffusion in solid ${ }^{4} \mathrm{He}$. When $U$ is large, the tunneling frequency $t_{i}$ for direct impurity-host exchanges is negligible, $t_{i} \sim t_{v}^{2} / U \ll t_{v}$, and the vacancy-assisted processes can dominate impurity motion with an effective tunneling rate $t_{i} \sim t_{v} a^{3} n_{v} \gg t_{v}^{2} / U$ (in this context, the asymmetry of the vacancy-assisted motion ${ }^{10}$ is not important). The impurity mean free path at $n_{v} \rightarrow 0$ is atomic while at $n_{v} \rightarrow N$ it is large and is determined by the scattering by other impurities or remaining upperstate host atoms. At $T>T_{c}$, the vacancy-assisted processes are responsible for impurity diffusion with

$$
D_{i} \sim \frac{t_{v} a^{2}}{\hbar} \frac{n_{v}}{N-n_{v}}
$$

After the vacancy system undergoes the superfluid transition (1), the impurity becomes a completely delocalized quasiparticle in the vacancy superfluid background similar to ${ }^{3} \mathrm{He}$ impurities in superfluid ${ }^{4} \mathrm{He}^{11}$. The effective mass of such quasiparticles at $T=0$ is $m_{i}^{*} \sim \hbar^{2} / 2 t_{v} a^{5} n_{v}$ and goes up with temperature with a decrease in density of the vacancy condensate. The interaction effects in this quasiparticle gas are negligible. At low temperatures, the impurities with density $n_{i}$ also undergo BEC with

$$
T_{c i} \simeq 6.62 t_{v} a^{5} n_{v} n_{i}^{2 / 3}=a^{3} n_{v}^{1 / 3} n_{i}^{2 / 3} T_{c}
$$

where $T_{c}$ is the temperature for the vacancy BEC (1). The emerging system with two condensates should exhibit properties similar to those of liquid ${ }^{3} \mathrm{He}-{ }^{4} \mathrm{He}$ mixtures with two condensates below the ${ }^{3} \mathrm{He}$ transition ${ }^{12}$. Since this BEC is based on the vacancy-assisted tunneling, the corresponding twocondensate system is different from the one considered in Ref. 9.

The band motion of the vacancies is possible when the impurity concentration $x_{i}=a^{3} n_{i}$ is low. At higher $x_{i}$, the vacancy motion is accompanied by the host-impurity permutations suppressing the band motion. This is 


\section{Optical Lattices: A New Type of Quantum Crystals?}

similar to the vacancy motion in solid ${ }^{3} \mathrm{He}$ with disordered spins. Then the vacancies autolocalize within the homogeneous domains of the size

$$
R=\left[\frac{\pi \hbar^{2}}{2 m^{*} N T\left[\left(x_{i}-1\right) \ln \left(1-x_{i}\right)-x_{i} \ln x_{i}\right]}\right]^{1 / 5}
$$

which are filled by particles in one hyperfine state (Nagaoka polarons). At large density of vacancies, $n_{v}^{1 / 3} R \gtrsim 1$, this leads to the decomposition of the system into macroscopic hyperfine domains. In contrast to the vacancydriven spin polarization of solid ${ }^{3} \mathrm{He}^{13}$, this transition takes place at fixed concentration of the zero-point vacancies and "polarization". This stationary decomposition is also different from the transient domains in Ref. 3.

Another issue is the sensitivity of the narrow-band particles to external fields. Since the energy cannot change by more than the bandwidth $\Delta$, the external field $\Omega(\mathbf{r})$ (e.g., the overall trapping potential $\Omega=\alpha r^{2} / 2$ ) localizes the particles in an area of size $\delta r \sim a \sigma, \sigma=\Delta /(\partial \Omega / \partial r) a$. In the momentum representation, $\frac{1}{2} \alpha r^{2}=\frac{1}{2} \hbar^{2} \alpha \nabla_{\mathbf{p}}^{2}$, the problem reduces to that for a particle with "mass" $1 / \alpha$ in potential $\epsilon(\mathbf{p})$. Near the band minima the motion is harmonic with the characteristic frequency $\omega_{0}^{*}=(2 t \alpha)^{1 / 2} a / \hbar$; $\omega_{0}^{*}$ is $\left(m / m^{*}\right)^{1 / 2}$ times lower than for the free particles. The quantization in potential $\Omega(\mathbf{r})$ is usually not important and the quasiclassical motion is unrestricted at large $\sigma$. When $\sigma \rightarrow 1$, even the classical motion becomes compressed towards the multiwell shells of thickness $\sigma a$ around the center of the trap. In experiment ${ }^{6}, \omega_{0}^{*} \sim 75\left(t / E_{r}\right)^{1 / 2} \mathrm{~Hz}, \sigma \gtrsim 100\left(t / E_{r}\right)$ and the shells are narrow when $t / E_{r} \lesssim 0.01$.

The inhomogeneity of the trap also leads to the non-uniform redistribution of particles ${ }^{5,14}$. If the change of the trapping potential from well to well is large, $\Delta / T \sigma \gg 1$, the shells with the lower energy are fully filled, have integer population, and become the MI. The rest of the shells will have non-integer population and resemble the vacancy liquid with a rather large density of vacancies. In experiment ${ }^{6}, \Delta / T \sigma \sim 10^{-10} / T$ (with $T$ in $\mathrm{K}$ ) seems to be small, meaning insignificant redistribution of particles between the shells. Even if this parameter were large, the system would represent a thick shell with $\sigma \gtrsim 100\left(t / E_{r}\right)$ of coupled well layers in the quasi-2D vacancy liquid state with the rest of the shells being the MI. This may actually increase the BEC temperature for the vacancy liquid since this liquid, though restricted to a lower number of shells, now has a higher density of vacancies.

In summary, we explored the analogy between the gases in optical lattices with non-integer occupation and large on-site interaction with QC. This explains experiment ${ }^{6}$ as a transition between the BEC and vacancy liquid states. The BEC for the vacancy liquid seems to be within experimental reach. The presence of unoccupied states provides a vacancy-assisted 


\section{A. E. Meyerovich}

mechanism for diffusion of hyperfine impurities and can lead to a spatial decomposition of the system into pure hyperfine components. The properties of the hyperfine mixture strongly depend on whether the system is above or below BEC for the vacancy liquid. At low temperatures one can observe the transition to the state with two - vacancy and impurity - condensates.

\section{ACKNOWLEDGMENTS}

The work is supported by NSF grants DMR-0077266 and INT-9724728.

\section{REFERENCES}

1. E. A. Cornell and C. E. Wieman, Rev. Mod. Phys. 74, 875 (2002).

2. A. J. Leggett, Rev. Mod. Phys. 73, 307 (2001); F. Dalfovo, S. Giorgini, L. P. Pitaevskii, and S. Stringari, Rev. Mod. Phys. 71, 463 (1999).

3. H. J. Lewandowski, D. M. Harber, D. L. Whitaker, and E. A. Cornell, Phys. Rev. Lett. 88, 070403 (2002); M. Ö. Oktel and L. S. Levitov, ibid, 88, 230403 (2002); J. N. Fuchs, D. M. Gangardt, and F. Laloë, ibid, 88, 230404 (2002); J. E. Williams, T. Nikuni, and C. W. Clark, ibid, 88, 230405 (2002); A. Kuklov and A. E. Meyerovich, Phys. Rev. A 66, 023607 (2002).

4. G. Raithel et al., Phys. Rev. Lett. 78, 630 (1997); T. Müller-Seydlitz et al., ibid., 1038 (1997); S. E. Hamann et al., ibid. 80, 4149 (1998); L. Guidoni et al., ibid, 79, 3363 (1997); K. I. Petsas, A. B. Coates, and G. Grynberg, Phys. Rev. A 50, 5173 (1994); I. H. Deutsch and P. S. Jessen, ibid, 57, 1972 (1998).

5. D. Jaksch, C. Bruder, J. I. Cirac, C. W. Gardiner, and P. Zoller, Phys. Rev. Lett. 81, 3108 (1998)

6. M. Greiner, O. Mandel, T. W. Hansch, and I. Bloch, Nature 415, 39 (2002).

7. A. F. Andreev, Defects and Surface Phenomena in Quantum Crystals, in: Quantum Theory of Solids, ed. I. M. Lifshits (Mir Publishers, Moscow, 1982) p. 11-69

8. P. Remeijer, S. C. Steel, R. Jochemsen, G. Frossati, and J. M. Goodkind, Sov.Phys. - Low Temp. Phys., 23, 438 (1997) [Fiz. Nizk. Temp. 23, 586 (1997)]; J. M. Goodkind, Phys. Rev. Lett. 89, 095301 (2002).

9. A.B. Kuklov and B.V. Svistunov, cond-mat/0205069 (2002).

10. O. A. Andreeva, K. O. Keshishev, and A. D. Savischev, Physica B: Cond. Matt. 284-288, 343 (2000).

11. E.P. Bashkin, and A.E. Meyerovich, Adv. Phys. 30, 1 - 92 (1981).

12. A.E.Meyerovich, in: Prog. Low Temp. Phys., ed. by D. F. Brewer (NorthHolland, Amsterdam, 1987), Vol. 11, pp. 1-73; A.E.Meyerovich, Spin-Polarized Phases of ${ }^{3} \mathrm{He}$, in: Helium Three, eds. W.P.Halperin and L.P.Pitaevski (Elsevier, Amsterdam) 1990, pp. 757 - 879.

13. A. F. Andreev, V. I. Marchenko, and A. E. Meyerovich, JETP Lett. 26, 36 (1977).

14. V. A. Kashurnikov, N. V. Prokof'ev, and B. V. Svistunov, Phys. Rev. A 66, 031601 (2002). 